\title{
Prediction of ovarian response in IVF/ICSI cycles
}

\author{
Alfredo Cortés-Vazquez ${ }^{1}$, Guillermo A. Goitia-Landeros ${ }^{1}$, Miguel A. Regalado ${ }^{1}$, Saúl R. León-Hernández ${ }^{2}$, Alfredo \\ L. Cortés-Algara ${ }^{1}$, Cindy Bandala ${ }^{3,4}$, Jesús D. Moreno-García ${ }^{1}$, Panagiotis Drakopoulos ${ }^{5,6}$ \\ ${ }^{1}$ Instituto de Seguridad Y Servicios Sociales de Los Trabajadores del Estado, Mexico City, Mexico \\ ${ }^{2}$ Investigation Support Unit, Instituto Nacional de Rehabilitación Luis Guillermo Ibarra Ibarra, Mexico City, \\ Mexico \\ ${ }^{3}$ Neuroscience Laboratory, Instituto Nacional de Rehabilitación Luis Guillermo Ibarra Ibarra, Mexico City, Mexico \\ ${ }^{4}$ Escuela Superior de Medicina, Instituto Politécnico Nacional, Mexico City, Mexico \\ ${ }^{5}$ Center for Reproductive Medicine, Universitair Ziekenhuis Brussel, Brussels, Belgium \\ ${ }^{6}$ Faculty of Medicine and Pharmacy, Department of Surgical and Clinical Science, Vrije Universiteit Brussel, \\ Belgium
}

\begin{abstract}
Objective: Our study aims to evaluate the various correlations between demographic, biochemical, ultrasound, and ovarian stimulation parameters with the percentage of mature oocytes in conventional stimulation for IVF/ICSI cycles in order to develop a predictive model to improve our understanding of the oocyte maturation process.

Methods: This is a retrospective cohort study; patients underwent conventional antagonist ovarian stimulation protocols for fresh IVF/ICSI cycles. A total of 256 IVF/ ICSI cycles were included. Age, antral follicle count (AFC), baseline serum follicle-stimulating hormone (FSH) levels, baseline serum luteinizing hormone (LH) levels, baseline serum estradiol (E2) levels, peak estradiol, P4 on hCG day, the body mass index (BMI), and stimulation length were measured. The variables were tested for correlations with the number of retrieved oocytes (\#RO) and the number of mature oocytes (\#MO). A backward stepwise regression was performed to identify the variables that correlated more strongly with percentage of mature oocytes (\%MO).

Results: A predictive equation was obtained with the variables that were not excluded in the model. $\% \mathrm{MO}=$ $72.700-0.910$ (Age) +0.979 (BMI) + 1.209 (Baseline serum LH) - 0.647 (Progesterone on human Chorionic Gonadotropin day).

Conclusions: We concluded that age, the BMI, baseline serum LH, and progesterone level on hCG day may predict $\%$ MO. Prospective studies are required to validate this predictive equation.
\end{abstract}

Keywords: Oocyte, Mature, Age, body mass index, progesterone, LH

\section{INTRODUCTION}

The world entered a new era in 1978, with the birth of Louise Brown. Since then, more than five million babies have been born worldwide through in vitro fertilization (IVF) techniques (van Loendersloot et al., 2014). This global trend was not caused by a recent infertility pandemic, but by increased access to IVF treatments. Differently from common belief, IVF does not guarantee success; between $38 \%$ and $49 \%$ of the couples have unsuccessful IVF cycles even after undergoing six IVF cycles (Malizia et al., 2009).

One of the strategies commonly used to increase pregnancy rate is to induce multiple follicles, to produce several mature oocytes and embryos subsequently. Oocyte maturation is essential because only metaphase II (MII) oocytes are injected in intracytoplasmic sperm injection (ICSI) cycles. MI oocytes that complete maturation in vitro exhibit lower fertilization rates. Only sporadic pregnancies were obtained following the transfer of embryos developed from MI oocytes that had matured in vitro (Strassburger et al., 2004; Nagy et al., 1996). The goal of oocyte cryopreservation is generally to freeze mature oocytes for later thawing and fertilization with IVF (Lee et al., 2013). However, $8.6 \%$ to $20 \%$ of the oocytes retrieved after controlled ovarian stimulation are metaphase I (MI) or immature at the germinal vesicle (GV) (Beall et al., 2010; Parrella et al., 2019), with total failure in oocyte maturation occurring occasionally, leading to no mature oocytes being produced (Beall et al., 2010). Successful fertilization depends on synchronic cytoplasmic and nuclear maturation (Pereira et al., 2016). There is evidence that an increased proportion of immature oocytes (GV and MI) diminish the ability of the MII sibling oocytes to be normally fertilized, resulting in a diminished number of good-quality embryos (Parrella et al., 2019).

Adverse effects from obesity on natural fecundity have been reported since the 1980s, with authors looking into the isolated impact of obesity on ICSI cycles. Statistically significant differences have been observed among women with different BMI. Women with a normal BMI had more MII oocytes than women with higher BMI (Esinler et al., 2008). Prospective observational cohort studies have reported a significant effect of the BMI on the number of mature oocytes. Women with a body mass index (BMI) greater than $25 \mathrm{~kg} / \mathrm{m}^{2}$ and final oocyte maturation with $0.2 \mathrm{mg}$ triptorelin had a significantly lower number of mature oocytes compared to women with a BMI below 25 $\mathrm{kg} / \mathrm{m}^{2}$ (Lainas et al., 2020).

This study evaluated the various associations between demographic, biochemical, ultrasound, and ovarian stimulation parameters with ovarian response, reflected in the number of mature oocytes obtained after conventional ovarian stimulation for IVF/ICSI.

\section{MATERIALS AND METHODS \\ Study Design}

After attaining approval from the institution's ethics committee, we performed a retrospective cohort analysis of 256 fresh IVF/ICSI cycles. The study included patients treated at the Reproductive Endocrinology Department of the Centro Médico Nacional 20 de Noviembre of Mexico City in 2018. Patient data sets were anonymized for analysis. The individuals included in this study underwent conventional ovarian stimulation protocol without adjuvant therapy. 


\section{Patients}

All patients underwent fresh IVF/ICSI cycles. Mild stimulation and social/medical freezing cycles were excluded.

\section{Protocol for Ovarian Stimulation}

Patients underwent conventional ovarian stimulation as described in the literature (Chalumeau et al., 2018). Ovarian stimulation was initiated with recombinant FSH ( $\mathrm{rFSH}$ ) (Gonal F, Merck-Serono, Switzerland) at a starting dose of 150-450 IU daily depending on the age, body mass index (BMI), and ovarian reserve (OR). Physicians subjectively chose the daily rFSH starting dose. A GnRH flexible antagonist protocol was used to achieve pituitary suppression (Cetrotide, cetrorelix $0.250 \mathrm{mg}$, Pierre Fabre, France) and recombinant human chorionic gonadotropin (rhCG) (Ovidrel, $250 \mathrm{mg}$, Merck-Serono, Italy) was administered to trigger ovulation when at least three leading follicles had reached a mean diameter of $18 \mathrm{~mm}$. Whenever necessary, dose adjustments of rFSH were performed according to the results of ovulation monitoring (ultrasound evaluation and estradiol). Data analysis revealed that 28 patients had late follicular phase progesterone elevation, and ten had ovarian hyperstimulation syndrome.

\section{Hormonal assays and evaluation of ovarian reserve}

Tests using the same method were performed in the same laboratory (Laboratorio Clínico, CMN 20 de Noviembre) for serum basal follicle-stimulating hormone (FSH), luteinizing hormone (LH), and estradiol (E2) between cycle days 2 or 3 . Serum progesterone (P4) and E2 concentration on the day of the trigger were measured. The samples were defined using an enzyme immunoassay (Axsym System, Advia Centaur; Siemens). To limit diurnal variations of hormone levels, all measurements were taken between 8 and 9 a.m.

Evaluation of ovarian reserve included antral follicle count (AFC) (2-9mm using a 2D 7.5 MHz probe, ClearVue 350, Philips, USA), baseline FSH, LH, and E2 concentrations.

\section{Oocyte retrieval and fertilization}

Oocytes were retrieved transvaginally 34-36 hours after rhCG administration. Oocytes were graded for maturity based on the morphological characteristics of the cumulus mass, corona radiata ooplasm and detached granulose membrane cells, presence or absence of a GV or the first polar body (PB) seen with a stereomicroscope, as described in the literature (Lin et al., 2003). Conventional
IVF was performed to fertilize mature and immature oocytes. The reasons for ICSI were male infertility and fertilization failure in conventional IVF. ICSI fertilized only MII oocytes. Only in the case of ICSI or unclear maturity, the oocytes were denuded using $85 \mathrm{IU} / \mathrm{ml}$ hyaluronidase and mechanical pipetting. The embryos were cultured to the blastocyst stage in sequential media.

\section{Endpoints}

Baseline demographic characteristics analyzed included age and BMI. Baseline IVF and ICSI cycle characteristics were FSH $(\mathrm{mIU} / \mathrm{ml})$, LH $(\mathrm{mIU} / \mathrm{ml})$, and E2 $(\mathrm{pg} / \mathrm{ml})$ levels at the start of the menstrual cycle. Conventional ovarian stimulation parameters included stimulation length (days), $\mathrm{E} 2$ and P4 concentration $(\mathrm{ng} / \mathrm{ml})$ on the day of trigger, total and mature oocytes retrieved, and percentage of mature oocytes.

\section{Statistical analysis}

Correlations between data extracted from the Reproductive Endocrinology Department database and the selected parameters were analyzed with Pearson's coefficient. A backward stepwise regression was performed to exclude parameters without statistical significance. Data analysis was performed on SPSS version 13.0 (SPSS Inc., USA).

\section{RESULTS}

A total of 256 IVF and ICSI cycles were included; baseline demographic characteristics are shown in Table 1. Mean age was $35.5 \pm 3.1$ years (24-44 years) and mean BMI was $26.8 \pm 4.0 \mathrm{~kg} / \mathrm{m}^{2}\left(18.6-34 \mathrm{~kg} / \mathrm{m}^{2}\right)$.

Table 1 shows the biochemical parameters, which effects are shown in Table 2. On average, seven oocytes were retrieved, five of which mature, meaning that $70 \%$ were MII oocytes. The number of retrieved oocytes was significantly correlated with age, AFC, FSH, and estradiol (E2) levels on trigger day, as seen in Table 3. It is essential to note that FSH and age were significantly correlated, while higher FSH levels and older age adversely affected the number of retrieved oocytes. Higher AFC and E2 levels on trigger day had a positive significant correlation with the number of retrieved oocytes. On step 2 of the backward stepwise regression, the number of mature oocytes had a significant positive correlation $(r=0.92, p=0.0001)$ with the total number of retrieved oocytes, in such a way that a higher number of retrieved oocytes corresponded to a greater number of mature oocytes as seen in Figure 1. After step 3, the percentage of mature oocytes was significantly correlated with the number of retrieved oocytes, BMI, AFC,

Table 1. Baseline patient demographic and IVF/ICS cycle characteristics

\begin{tabular}{|l|c|}
\hline \multicolumn{1}{|l|}{ Table 1. Baseline patient demographic and IVF/ICS cycle characteristics } \\
\hline & $(\mathrm{n}=256)$ \\
\hline Stimulation length (days) & $9.80 \pm 1.34$ \\
\hline BMI $\left(\mathrm{Kg} / \mathrm{m}^{2}\right)$ & $26.80 \pm 4.0$ \\
\hline Age $($ years $)$ & $35.50 \pm 3.10$ \\
\hline AFC & $13.87 \pm 8.37$ \\
\hline Baseline serum FSH $(\mathrm{mIU} / \mathrm{ml})$ & $6.82 \pm 3.21$ \\
\hline Baseline serum LH $(\mathrm{mIU} / \mathrm{ml})$ & $3.97 \pm 2.16$ \\
\hline Peak E2 $(\mathrm{pg} / \mathrm{ml})$ & $3181.69 \pm 6017.52$ \\
\hline P4 on hCG day $(\mathrm{ng} / \mathrm{ml})$ & $1.60 \pm 6.12$ \\
\hline Baseline serum E2 $(\mathrm{pg} / \mathrm{ml})$ & $65.9077 \pm 106.98$ \\
\hline
\end{tabular}

Data are expressed as mean \pm standard deviation. AFC $=$ antral follicle count, $B M I=$ body mass index, $F S H=$ follicle stimulating hormone, LH= luteinizing hormone, E2= estradiol, P4=progesterone, hCG= human chorionic gonadotropin. 
Table 2. Ovarian stimulation cycle outcomes

\begin{tabular}{|l|c|}
\hline & $(n=256)$ \\
\hline Oocytes retrieved $(n)$ & $7.01 \pm 4.44$ \\
\hline Mature oocytes $(n)$ & $5.0 \pm 3.59$ \\
\hline Mature oocytes $(n)$ & $70.31 \pm 21.08$ \\
\hline
\end{tabular}

Data are expressed as mean \pm SD

Table 3. Correlations between oocytes retrieved

\begin{tabular}{|l|c|c|}
\hline Parameter & Coefficient r \\
\hline Age & -0.19 & 0.002 \\
\hline AFC & 0.42 & 0.0001 \\
\hline Baseline serum FSH (mIU/ml) & -0.25 & 0.0001 \\
\hline Peak E2 & 0.38 & 0.0001 \\
\hline BMI & -0.004 & 0.94 \\
\hline Stimulation length & 0.04 & 0.50 \\
\hline Baseline serum LH (MIU/ml) & -0.06 & 0.32 \\
\hline P4 on hCG day & -0.03 & 0.58 \\
\hline Baseline serum E2 & 0.00 & 0.99 \\
\hline
\end{tabular}

$\mathrm{AFC}=$ antral follicle count, $\mathrm{FSH}=$ follicle stimulating hormone, $\mathrm{LH}=$ luteinizing hormone, $\mathrm{E} 2=$ estradiol, $\mathrm{P} 4=\mathrm{progesterone}$, hCG $=$ human chorionic gonadotropin.

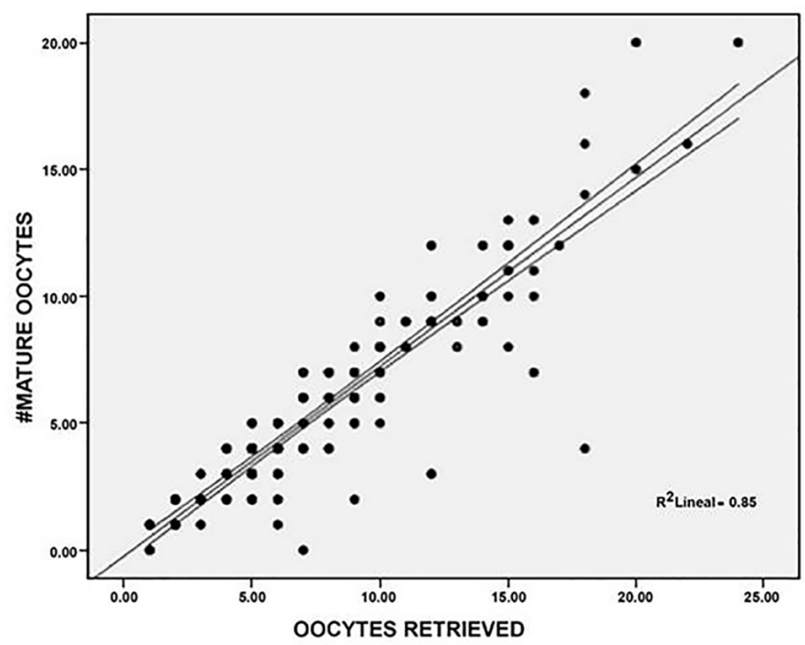

Figure 1. Correlation between number of retrieved oocyte and number of mature oocytes. \#Mature oocyte= number of mature oocytes

and progesterone (P4) levels on trigger day. The process was carried out until all variables were excluded in the $6^{\text {th }}$ step, at which time an equation to predict \% MO was developed.

It is essential to consider the fact that the constant 72.700 was also significant $(p=0001)$, in it a condition required for the model to be valid. Our model was potent to predict the number of retrieved oocytes, the number of mature oocytes, and \%OM.

Table 4 shows the variables excluded with the backward stepwise regression. It is crucial to understand that when patients were categorized according to age ( $<36$ years old or $>36$ years old), there were relevant differences in AFC, FSH levels, baseline LH level and, above all, in the number of retrieved oocytes, number of mature oocytes, and \%MO
(Table 4). When patients were categorized according to the BMI, differences were seen in FSH levels and AFC, but mainly on the number of mature oocytes and even more on \%MO. However, they did not differ in the number of retrieved oocytes.

\section{DISCUSSION}

Around $2 \%$ of women have primary infertility, and approximately $10 \%$ of women have secondary infertility, according to a World Health Organization survey (Nisal et al., 2020). Personalized assisted reproductive treatments help to reduce the financial burden that these treatments involve. Some studies have shown that dosages derived from predictive models are nearly $40 \%$ lower (Nisal et al., 2020) than the dosages prescribed in IVF clinics, with greater numbers of mature follicles. Patient-tailored care decreases the number of daily tests and increases patient satisfaction.

Patients with low oocyte maturity or an inability to retrieve mature oocytes have overwhelming prognoses on account of having fewer embryos for transfer. In our study, we found that baseline LH levels had a significant impact on the percentage of MII oocytes. There is evidence in cattle that $\mathrm{LH}$ regulates the expression of cell cyclerelated transcripts such as STMN1, BCAR3, ESCO2, PTTG1, or NCAPG, which are associated with DNA stabilization, oogenesis, folliculogenesis and, most notably, with the acquisition of competence (Sirard, 2016). Cell cycle regulation and function is a complex process, regulated by numerous genes, such as cyclin B1 or STMN1, which exhibit lower levels of mRNA in cetrorelix-treated cattle (Sirard, 2016). Therefore, the suppression of baseline LH during that period might reduce the quality of the oocytes.

We found that age negatively affected the percentage of mature oocytes, more specifically in individuals older than 36 years with a normal BMI. Below that threshold, age did not have an impact on healthy women irrespective of ovarian reserve. Other authors have reached the same conclusions and found no correlation between AFC and oocyte competence in women younger than 35 years (López 


\begin{tabular}{|c|c|c|c|c|c|c|}
\hline \multirow{3}{*}{$\begin{array}{l}\text { Model } \\
2\end{array}$} & \multirow{3}{*}{$\begin{array}{c}\text { Beta } \\
\text { Baseline serum FSH }\end{array}$} & \multirow{3}{*}{$\frac{\mathbf{t}}{-.010^{\mathrm{a}}}$} & \multirow{3}{*}{$\begin{array}{l}\text { Sig. } \\
-.134\end{array}$} & \multirow{3}{*}{$\begin{array}{c}\begin{array}{c}\text { Partial } \\
\text { Correlation }\end{array} \\
.893\end{array}$} & \multirow{2}{*}{\multicolumn{2}{|c|}{$\begin{array}{c}\text { Collinearity statistic } \\
\text { Tolerance }\end{array}$}} \\
\hline & & & & & & \\
\hline & & & & & -.009 & .713 \\
\hline 3 & $\begin{array}{l}\text { Baseline serum FSH } \\
\text { Stimulation duration }\end{array}$ & $\begin{array}{l}-.011^{\mathrm{b}} \\
-.024^{\mathrm{b}}\end{array}$ & $\begin{array}{l}-.144 \\
-.377\end{array}$ & $\begin{array}{l}.885 \\
.707\end{array}$ & $\begin{array}{l}-.009 \\
-.025\end{array}$ & $\begin{array}{l}.714 \\
.974\end{array}$ \\
\hline 4 & $\begin{array}{l}\text { Baseline serum FSH } \\
\text { Stimulation duration } \\
\text { Peak } E_{2}\end{array}$ & $\begin{array}{l}-.017^{c} \\
-.020^{c} \\
-.055^{c} \\
\end{array}$ & $\begin{array}{l}-.230 \\
-.321 \\
.844 \\
\end{array}$ & $\begin{array}{l}.818 \\
.749 \\
.400\end{array}$ & $\begin{array}{l}-.015 \\
-.021 \\
.055\end{array}$ & $\begin{array}{l}.721 \\
.978 \\
.910 \\
\end{array}$ \\
\hline 5 & $\begin{array}{l}\text { Baseline serum FSH } \\
\text { Stimulation duration } \\
\text { Peak } E_{2}\end{array}$ & $\begin{array}{l}-.006^{d} \\
-.021^{d} \\
-.055^{d} \\
\end{array}$ & $\begin{array}{l}-.083 \\
-.334 \\
.852 \\
\end{array}$ & $\begin{array}{l}.934 \\
.739 \\
.395\end{array}$ & $\begin{array}{l}-.005 \\
-.022 \\
.056\end{array}$ & $\begin{array}{l}.741 \\
.978 \\
.910\end{array}$ \\
\hline 6 & $\begin{array}{l}\text { Baseline serum FSH } \\
\text { Stimulation duration } \\
\text { Peak } E_{2} \\
\text { Baseline serum } E_{2} \\
\text { AFC }\end{array}$ & $\begin{array}{l}-.023^{\mathrm{e}} \\
-.022^{\mathrm{e}} \\
-.071^{\mathrm{e}} \\
-.058^{\mathrm{e}} \\
-.075^{\mathrm{e}}\end{array}$ & $\begin{array}{l}-.330 \\
-.355 \\
1.141 \\
.927 \\
1.179\end{array}$ & $\begin{array}{l}.742 \\
.723 \\
.255 \\
.355 \\
.240\end{array}$ & $\begin{array}{l}-.022 \\
-.023 \\
.074 \\
.060 \\
.077\end{array}$ & $\begin{array}{l}.776 \\
.978 \\
.983 \\
.981 \\
.943\end{array}$ \\
\hline
\end{tabular}

a. Predictive variables in the model: (Constant), Baseline serum E2, P4 on hCG day, peak E2, BMI, Stimulation duration, Age, AFC, baseline serum LH

b. Predictive variables in the model: (Constant), Baseline serum E2, P4 on hCG day, peak E2, BMI, Age, AFC, baseline serum $\mathrm{LH}$

c. Predictive variables in the model: (Constant), Baseline serum E2, P4 on hCG day, BMI, Age, AFC, baseline serum LH

d. Predictive variables in the model: (Constant), P4 on hCG day, BMI, Age, AFC, baseline serum LH

e. Predictive variables in the model: (Constant), P4 on hCG day, BMI, Age, baseline serum LH

f. Dependent variables: \% mature oocytes

Martín et al., 2018). However, not all populations seem to behave in the same way, and some authors observed that the rate of immature oocyte was not proportional to female patient age. Indeed, the highest rates of immature oocytes were seen in women older than 41 years, followed women younger than 30 years old (Lee et al., 2012).

It is essential to discuss and encourage future research on the effects of stimulation on \%MO. We observed that stimulation length did not correlate with \%MO, as described by other authors, who found that delaying oocyte maturation trigger by 24 hours did not affect the number of MII oocytes (Davar et al., 2017), although other clinicians have different opinions and some concluded that delaying the administration of hCG by 24 hours with progesterone levels of less than $1 \mathrm{ng} / \mathrm{ml}$ might yield a higher number of MII oocytes (Vandekerckhove et al., 2014). Unquestionably, the ovarian reserve plays a pivotal role in the outcome of stimulation. At the same time, normal responders had significantly lower maturation rates with less than six stimulation days and low responders had similar stimulation days with similar maturation rates with either short or long stimulation length, as observed by other authors (Yang et al., 2019).

The role of progesterone in ovarian stimulation cycles and its effect on the maturation process must be further recognized. Our study found a negative effect of progesterone on hCG day and \%MO. Tanada et al. (2017) observed the same effect and found that premature late follicular phase progesterone increases above $1.3 \mathrm{ng} / \mathrm{ml}$ might adversely affect the oocyte maturation process.

Recently, Simon et al. (2019) evaluated the progesterone to mature oocytes index (PMOI) and found that lower PMOI was associated with increased live births and a statistically significant increased number of mature oocytes. They also found that the PMOI was increased mainly in low ovarian response, which was evaluated through the Ovarian Sensitivity Index (OSI). Likewise, it should be noted that high blood progesterone levels on the day of ovulation trigger may reflect either many follicles with proper follicular fluid progesterone levels or a few follicles with high follicular fluid progesterone levels (Grin et al., 2018).

Huang et al. (2016) published a retrospective study with 4236 fresh IVF cycles, in which progesterone levels higher than $2.0 \mathrm{ng} / \mathrm{ml}$ were associated with a reduction on the rate of top quality embryos. It might be worth encouraging research to establish a progesterone threshold in humans in which \%MO is maximal. Also, it is essential to address the highly important role of cumulus cells in oocyte health; increased cumulus cell apoptosis is followed by impaired oocyte maturation and lower fertilization rates (Dumesic et al., 2015). Thus, ovarian stimulation protocols that apparently improve cumulus cells activity might have a remarkable effect on oocyte health, consequently increasing the proportion of oocytes that develop into healthy embryos and eventually yield live births. This is particularly relevant, since approximately less than $7 \%$ of the oocytes retrieved by IVF generate a healthy embryo (Dumesic et al., 2015).

We found a positive correlation between the BMI and $\%$ MO. However, other authors have not observed this positive correlation. They found that overweight and obese women had altered oocyte morphology and lower fertilization rates, particularly when aged less than 35 years (Dumesic et al., 2015). Some studies demonstrated that hyperglycemia and high lipid concentrations disrupt somatic cell-oocyte signaling, leading to impaired growth and delayed maturation (Dumesic et al., 2015; Varghese et al., 2011). Primarily high lipid concentrations were associated with germinal vesicle breakdown inhibition, which prevents the oocyte from proceeding beyond the arrested prophase 1 stage (Varghese et al., 2011). In addition to obesity, it is crucial to keep in mind some infertility-associated pathologies, such as polycystic ovary syndrome (PCOS), in which higher androgen levels such as androstenedione are observed, a steroid hormone linked to oocyte maturation inhibition and spindle assembly prevention, which alters chromosomal alignment in oocytes (Tarumi et al., 2012), 
best known as maturation failure type 2 (Beal et al., 2010). Lainas et al. (2020) conducted a prospective observational cohort study, in which the BMI significantly affected the number of mature oocytes. Women with a BMI higher than $25 \mathrm{~kg} / \mathrm{m}^{2}$ had significantly fewer mature oocytes when compared to women with a BMI below $25 \mathrm{~kg} / \mathrm{m}^{2}$ (Esinler et al., 2008; Lainas et al., 2020). Above all, it is crucial to note that although there is no consensus about the optimal dose for $\mathrm{GnRHa}$ in trigger protocols, the most popular dose of $\mathrm{GnRH}$ agonist to trigger final oocyte maturation is $0.2 \mathrm{mg}$ triptorelin (Engmann et al., 2016). Consequently, a dose adjustment in the oocyte maturation trigger protocol for obese women is suggested, mainly when a GnRH agonist trigger is indicated (Lainas et al., 2020). We hypothesize that obesity increases the distribution volume and attenuates the effects of the continuously elevated $\mathrm{FSH}$ levels during ovarian stimulation, therefore reducing the quantity of precursor steroids generated and the ability of the ovary to convert them into estrogen.

To our knowledge, this is the first model to predict $\% O M$. It is valuable to know in advance whether a patient might have low \%OM, so that further procedures such as rescue IVM or dual trigger might be applied to increase the number of oocytes that eventually develop into viable embryos. There are several and very promising approaches to enhance ovarian stimulation protocols and lower serum progesterone levels, including the administration of corticosteroids in the follicular phase, reduction of $\mathrm{FSH}$ stimulation intensity towards the end of stimulation, use of coriofollitropin alpha, and stimulation length reduction (Lawrenz et al., 2018). The weaknesses of our study include the way oocyte maturity was assessed, the fact that it is a retrospective study, and the limited number of patients. Additional prospective studies are required to validate the results published herein.

\section{CONCLUSIONS}

The percentage of mature oocytes retrieved after conventional ovarian stimulation was strongly correlated with baseline serum LH and P4 on hCG day levels, age, and the BMI. Further prospective studies must be performed to confirm and validate these findings.

\section{CONFLICT OF INTEREST}

None.

\section{Corresponding author}

Alfredo Cortés-Vazquez

Instituto de Seguridad Y Servicios Sociales de Los Trabajadores del Estado

Mexico City, Mexico

Email: alfredo.cortes.vazquez@gmail.com

\section{REFERENCES}

Beall S, Brenner C, Segars J. Oocyte maturation failure: a syndrome of bad eggs. Fertil Steril. 2010;94:2507-13. PMID: 20378111 DOI: 10.1016/j.fertnstert.2010.02.037

Chalumeau C, Moreau J, Gatimel N, Cohade C, Lesourd $\mathrm{F}$, Parinaud J, Leandri R. Establishment and validation of a score to predict ovarian response to stimulation in IVF. Reprod Biomed Online. 2018;36:26-31. PMID: 29111311 DOI: $10.1016 /$ j.rbmo.2017.09.011

Davar R, Naghshineh E, Neghab N. The effect of 24 hours delay in oocyte maturation triggering in IVF/ICSI cycles with antagonist protocol and not-elevated progesterone: A randomized control trial. Int J Reprod Biomed. 2017;15:4416. PMID: 29177246 DOI: 10.29252/ijrm.15.7.441
Dumesic DA, Meldrum DR, Katz-Jaffe MG, Krisher RL, Schoolcraft WB. Oocyte environment: follicular fluid and cumulus cells are critical for oocyte health. Fertil Steril. 2015;103:30316. PMID: 25497448 DOI: 10.1016/j.fertnstert.2014.11.015

Engmann L, Benadiva C, Humaidan P. GnRH agonist trigger for the induction of oocyte maturation in GnRH antagonist IVF cycles: a SWOT analysis. Reprod Biomed Online. 2016;32:27485. PMID: 26803205 DOI: 10.1016/j.rbmo.2015.12.007

Esinler I, Bozdag G, Yarali H. Impact of isolated obesity on ICSI outcome. Reprod Biomed Online. 2008; 17:583-7. PMID: 18854116 DOI: $10.1016 / S 1472-6483(10) 60249-0$

Grin L, Mizrachi Y, Cohen O, Lazer T, Liberty G, Meltcer S, Friedler S. Does progesterone to oocyte index have a predictive value for IVF outcome? A retrospective cohort and review of the literature. Gynecol Endocrinol. 2018;34:638-43. PMID: 29373930 DOI: 10.1080/09513590.2018.1431772

Huang B, Ren X, Wu L, Zhu L, Xu B, Li Y, Ai J, Jin L. Elevated Progesterone Levels on the Day of Oocyte Maturation May Affect Top Quality Embryo IVF Cycles. PLoS One. 2016;11:e0145895. PMID: 26745711 DOI: 10.1371/journal.pone.0145895

Lainas GT, Lainas TG, Sfontouris IA, Venetis CA, Bosdou JK, Chatzimeletiou A, Grimbizis GF, Tarlatzis BC, Kolibianakis EM. Association between body mass index and oocyte maturation in patients triggered with GnRH agonist who are at high risk for severe ovarian hyperstimulation syndrome: an observational cohort study. Reprod Biomed Online. 2020;40:168-75. PMID: 31839394 DOI: 10.1016/j.rbmo.2019.10.006

Lawrenz B, Labarta E, Fatemi H, Bosch E. Premature progesterone elevation: targets and rescue strategies. Fertil Steril. 2018;109:577-82. PMID: 29653703 DOI: 10.1016/j.fertnstert.2018.02.128

Lee HJ, Jee BC, Suh CS, Kim SH, Moon SY. Oocyte maturity in relation to woman's age in in vitro fertilization cycles stimulated by single regimen. Yonsei Med J. 2012; 53:1815. PMID: 22187250 DOI: 10.3349/ymj.2012.53.1.181

Lee JA, Barritt J, Moschini RM, Slifkin RE, Copperman AB. Optimizing human oocyte cryopreservation for fertility preservation patients: should we mature then freeze or freeze then mature? Fertil Steril. 2013;99:1356-62. PMID: 23266213 DOI: $10.1016 /$ j.fertnstert.2012.11.042

López Martín MJ, García D, Vassena R, Rodríguez A. Oocyte developmental competence is independent of ovarian reserve in women younger than 35 years. Reprod Biomed Online. 2018; 37:677-84.

Lin YC, Chang SY, Lan KC, Huang HW, Chang CY, Tsai MY, Kung FT, Huang FJ. Human oocyte maturity in vivo determines the outcome of blastocyst development in vitro. J Assist Reprod Genet. 2003;20:506-12. PMID: 15035550 DOI: $10.1023 / \mathrm{b}$ :jarg.0000013651.37866.0c

Malizia BA, Hacker MR, Penzias AS. Cumulative live-birth rates after in vitro fertilization. N Engl J Med. 2009;360:23643. PMID: 19144939 DOI: 10.1056/NEJMoa0803072

Nagy ZP, Cecile J, Liu J, Loccufier A, Devroey P, Van Steirteghem $A$. Pregnancy and birth after intracytoplasmic sperm injection of in vitro matured germinal-vesicle stage oocytes: case report. Fertil Steril. 1996;65:1047-50. PMID: 8612833 DOI: 10.1016/S0015-0282(16)58285-5 
Nisal A, Diwekar U, Bhalerao V. Personalized medicine for in vitro fertilization procedure using modeling and optimal control. J Theor Biol. 2020;487:110105. PMID: 31809718 DOI: $10.1016 /$ j.jtbi.2019.110105

Parrella A, Irani M, Keating D, Chow S, Rosenwaks Z, Palermo GD. High proportion of immature oocytes in a cohort reduces fertilization, embryo development, pregnancy and live birth rates following ICSI. Reprod Biomed Online. 2019;39:580-7. PMID: 31455582 DOI: 10.1016/j.rbmo.2019.06.005

Pereira N, Neri QV, Lekovich JP, Palermo GD, Rosenwaks $Z$. The role of in-vivo and in-vitro maturation time on ooplasmic dysmaturity. Reprod Biomed Online. 2016; 32:401-6. PMID: 26896430 DOI: $10.1016 / \mathrm{j}$. rbmo.2016.01.007

Simon C, Branet L, Moreau J, Gatimel N, Cohade C, Lesourd F, Parinaud J, Leandri R. Association between progesterone to number of mature oocytes index and live birth in GnRH antagonist protocols. Reprod Biomed Online. 2019;38:901-7. PMID: 30952493 DOI: 10.1016/j. rbmo.2019.01.009

Sirard MA. Somatic environment and germinal differentiation in antral follicle: The effect of FSH withdrawal and basal LH on oocyte competence acquisition in cattle. Theriogenology. 2016;86:54-61. PMID: 27158126 DOI: $10.1016 / \mathrm{j}$.theriogenology.2016.04.018

Strassburger D, Friedler S, Raziel A, Kasterstein E, Schachter M, Ron-EI R. The outcome of ICSI of immature MI oocytes and rescued in vitro matured MII oocytes. Hum Reprod. 2004;19:1587-90. PMID: 15131077 DOI: 10.1093/humrep/deh236
Tanada MS, Yoshida IH, Santos M, Berton CZ, Souto E, Carvalho WP, Cordts EB, Barbosa CP. The impact of high progesterone levels on the day of HCG administration in assisted human reproduction treatments. JBRA Assist Reprod. 2018;22:99-101. PMID: 29345445 DOI: 10.5935/1518-0557.20180020

Tarumi W, Tsukamoto S, Okutsu Y, Takahashi N, Horiuchi T, Itoh MT, Ishizuka B. Androstenedione induces abnormalities in morphology and function of developing oocytes, which impairs oocyte meiotic competence. Fertil Steril. 2012;97:46976. PMID: 22289288 DOI: 10.1016/j.fertnstert.2011.11.040

van Loendersloot L, Repping S, Bossuyt PM, van der Veen $\mathrm{F}$, van Wely $\mathrm{M}$. Prediction models in in vitro fertilization; where are we? A mini review. J Adv Res. 2014;5:295-301. PMID: 25685496 DOI: 10.1016/j.jare.2013.05.002

Vandekerckhove F, Gerris J, Vansteelandt S, De Baerdemaeker A, Tilleman K, De Sutter P. Delaying the oocyte maturation trigger by one day leads to a higher metaphase II oocyte yield in IVF/ICSI: a randomised controlled trial. Reprod Biol Endocrinol. 2014;12:31. PMID: 24758641 DOI: $10.1186 / 1477-7827-12-31$

Varghese AC, Ly KD, Corbin C, Mendiola J, Agarwal A. Oocyte developmental competence and embryo development: impact of lifestyle and environmental risk factors. Reprod Biomed Online. 2011;22:410-20. PMID: 21388885 DOI: $10.1016 /$ j.rbmo.2010.11.009

Yang YC, Li YP, Pan SP, Chao KH, Chang CH, Yang JH, Chen $\mathrm{SU}$. The different impact of stimulation duration on oocyte maturation and pregnancy outcome in fresh cycles with $\mathrm{GnRH}$ antagonist protocol in poor responders and normal responders. Taiwan J Obstet Gynecol. 2019; 58:471-6. PMID: 31307735 DOI: 10.1016/j.tjog.2019.05.007 